\title{
A avaliação clínica do idoso que cai
}

\author{
Clinical assessment of the elderly who fall
}

La evaluación médica del adulto mayor que se cae

\author{
Emylucy M. P. Paradela*
}

\section{Resumo}

O envelhecimento pode provocar alterações que impactam negativamente o equilíbrio e a marcha, aumentando assim a vulnerabilidade dos idosos às quedas. Muitos idosos não relatam dificuldades para andar durante uma consulta médica, alguns não referem já terem caído por desconhecerem intervenções que possam minimizar o problema. A instabilidade postural é considerada uma síndrome geriátrica com sinais e sintomas próprios e com etiologia multifatorial. Uma queda em um indivíduo idoso pode ser considerada um evento sentinela ou um sinal de alerta anunciando um declínio funcional, um quadro infeccioso assintomático, o efeito adverso de um medicamento ou uma patologia subjacente sem sinais e sintomas clássicos. O exame físico de um idoso começa antes mesmo de sua entrada no consultório. O médico deve estar atento à maneira como o paciente se levanta da cadeira e anda, se precisa de ajuda para se levantar ou se usa algum aparelho para auxiliar a marcha. A avaliação do equilíbrio e das alterações da marcha, assim como a investigação da ocorrência de quedas em idosos, deve ser muito cuidadosa; durante a entrevista clínica o médico deve perguntar diretamente sobre problemas do equilíbrio, marcha e ocorrência de quedas, assim como conhecer as circunstâncias em que ocorreram. Habitualmente as quedas são consequências de distúrbios do equilíbrio e da marcha, enquanto algumas delas são típicas de patologias específicas. Por isto, a observação cuidadosa da marcha pode ajudar o médico a elaborar hipóteses diagnósticas mais prováveis a fim de propor intervenções preventivas.

Descritores: Envelhecimento; Acidentes por quedas; Marcha; Equilíbrio postural.

\begin{abstract}
Aging can cause changes that negatively impact the balance as well as the gait, thereby increasing the vulnerability of the elderly to falls. Many seniors do not report difficulty in walking during a medical appointment; some do not mention having already fallen because they are not aware of interventions that can mitigate the problem. Postural instability is considered a geriatric syndrome with signs and symptoms of its own multifactorial etiology. A fall in an elderly individual can be considered a sentinel event or a warning sign announcing a functional decline, an asymptomatic infection, the adverse effect of a drug, or an underlying pathology without classic signs and symptoms. Physical examination of an elderly begins even before entering the office. The physician should be alert to the way they rise from the chair and walk, if they need help to stand or use any device to assist walking. The assessment of balance and gait alterations as well as the investigation of the occurrence of falls in the elderly should be made very carefully: during the clinical interview, the doctor should directly ask about issues involving balance, gait and the occurrence of falls and find out the circumstances in which they occurred. Usually falls are a consequence of balance and gait disturbances and some of them are typical of specific pathologies. Therefore, careful observation of the gait can help the physician to develop diagnostic hypotheses most likely to be correct in order to propose preventive interventions.
\end{abstract}

Keywords: Aging; Accidental falls; Gait; Postural balance.

\section{Resumen}

El envejecimiento puede provocar alteraciones que impactan negativamente el equilibrio y la marcha, aumentando así la vulnerabilidad de los adultos mayores a las caídas. Durante una consulta médica, muchos adultos mayores 
no mencionan sus dificultades para andar, algunos no se refieren a haberse caído ya porque desconocen las intervenciones que puedan minimizar el problema. La inestabilidad postural es considerada un síndrome geriátrico con señales, síntomas propios y con etiología multifactorial. Una caída en un adulto mayor puede considerarse un evento centinela o una señal de alerta que anuncia un declive funcional, un cuadro infeccioso asintomático, o el efecto adverso de un medicamento o una patología subyacente sin señales y síntomas clásicos. El examen físico de un adulto mayor comienza incluso antes de su ingreso en el consultorio. El médico debe estar atento a la manera en la que él se levanta de la silla y anda, si necesita ayuda para levantarse o si usa algún aparato para ayudar a la marcha. La evaluación del equilibrio y de las alteraciones de la marcha, así como la investigación de la ocurrencia de caídas en adultos mayores debe ser muy cuidadosa; durante la entrevista clínica el médico debe preguntar abiertamente sobre los problemas de equilibrio, marcha y ocurrencia de caídas, así como conocer las circunstancias en que acontecieron. Habitualmente, las caídas son consecuencias de perturbaciones del equilibrio y de la marcha, algunas de éstas son típicas de patologías específicas, por esto, la observación cuidadosa de la marcha puede ayudar al médico a elaborar hipótesis diagnósticas más probables a fin de proponer intervenciones preventivas.

Palabras clave: Envejecimiento; Accidentes por caídas; Marcha; Equilibrio postural.

\section{Introdução}

Para andarmos com segurança, sem cairmos aos pequenos tropeços, necessitamos de uma boa coordenação motora, equilíbrio preservado e boa acuidade visual e auditiva, assim como articulações e músculos íntegros e eficientes. ${ }^{1,2}$

A instabilidade postural é considerada uma síndrome geriátrica com sinais e sintomas próprios e com etiologia multifatorial. Os distúrbios do equilíbrio e da marcha devem ser investigados cuidadosamente, pois os idosos que caminham de maneira insegura, assim como aqueles que já caíram, têm maior chance de sofrer quedas, com todas as consequências trágicas advindas destes episódios.

Muitos idosos não relatam dificuldades para deambular durante uma consulta médica. Alguns não referem já terem caído por desconhecerem intervenções que possam reverter ou minimizar o problema. O médico deve perguntar diretamente sobre problemas do equilíbrio, marcha e ocorrência de quedas, assim como conhecer as circunstâncias em que ocorreram.

Uma queda em um indivíduo idoso pode ser considerada um evento sentinela, um sinal de alerta anunciando um declínio funcional, um quadro infeccioso assintomático, o efeito adverso de um medicamento ou uma patologia subjacente sem sinais e sintomas clássicos.

\section{Avaliação clínica}

A avaliação do equilíbrio e das alterações da marcha, assim como a investigação da ocorrência de quedas em idosos, deve ser muito cuidadosa; durante a entrevista clínica o médico deve perguntar diretamente sobre problemas do equilíbrio, marcha e ocorrência de quedas.

Alguns exemplos de perguntas a serem utilizadas na anamnese: Houve alguma alteração na sua maneira de andar nos últimos meses? Tem tido algum desequilíbrio com a sensação de que vai cair? Tem medo de cair? Caiu alguma vez nos últimos seis meses? Restringiu suas atividades habituais por causa de uma queda ou do medo de cair? Caso tenha caído, em que lugar aconteceu? Houve perda da consciência? Como a queda ocorreu? Houve alguma modificação na medicação usada habitualmente?

O exame físico de um idoso começa antes mesmo de sua entrada no consultório. O médico deve estar atento à maneira como o paciente se levanta da cadeira e anda, se precisa de ajuda para se levantar ou se usa algum aparelho para auxiliar a marcha.

Um exame físico completo deve ser realizado, assim como a avaliação do equilíbrio e da marcha. A avaliação de cada aparelho e sistema deve ser cuidadosamente realizada.

Aparelho cardiovascular: na avaliação cardíaca, o médico deve estar atento à presença de arritmias e hipotensão ortostática (HO). A HO pode ser sintomática, com tonteira ou sensação de cabeça vazia, ou assintomática. O diagnóstico é feito medindo-se a pressão arterial (PA) nos dois braços em decúbito dorsal, assim como a frequência cardíaca (FC); depois, o paciente deve ficar sentado e após três minutos a PA é medida novamente no braço que apresentou a medida 
mais elevada. Por último, após três minutos com o paciente em pé, a PA é medida novamente. A redução da PA sistólica em $20 \mathrm{mmHg}$ ou mais, assim como um aumento da frequência cardíaca em dez batimentos por minuto após a alteração postural, confirma o diagnóstico de $\mathrm{HO}^{3}$

Outras causas cardíacas comuns para quedas em idosos que devem ser investigadas: estenose aórtica, insuficiência coronariana e isquemia vertebrobasilar.

Sistema neurológico: durante o exame neurológico o médico deve estar atento às principais alterações que levam os idosos a cair, como a doença de Parkinson, sequelas de acidentes vasculares encefálicos, demências, hidrocefalia de pressão normal, doenças vestibulares e neuropatias periféricas. ${ }^{4}$

Sistema musculoesquelético: durante o exame físico o médico deve estar atento às principais alterações musculoesqueléticas que levam os idosos a cair, como a hipotrofia muscular, patologias articulares, alterações nos pés e deformidades da coluna vertebral. ${ }^{5}$

Outras causas de quedas em idosos que devem ser investigadas: descondicionamento físico, efeitos colaterais de drogas, deficiência de vitamina B12, depressão, delirium, edema dos membros inferiores, doenças vestibulares, gota, doenças pulmonares, doenças crônicas descompensadas e deficit visual. ${ }^{6}$

Complementando o exame físico, o médico deve avaliar o equilíbrio e a marcha com a simples visualização ou utilizando testes padronizados como o Get Up and Go, o teste de Tinetti e o teste de alcance funcional. ${ }^{7-9}$

O teste Get Up and Go pode ser útil para avaliar a mobilidade e o equilíbrio. É amplamente utilizado por ser de fácil aplicação. Consiste em observar o paciente após pedir: "Levante-se da cadeira, ande três metros, retorne e sente-se." Durante o teste observa-se a capacidade muscular dos membros inferiores de levantar de uma cadeira sem apoio, o equilíbrio e a posição dos pés e braços durante a marcha, assim como a estabilidade do corpo.

O teste de Tinetti consiste em uma escala com 16 tarefas que são avaliadas por meio da observação do examinador. São atribuídos pontos de 0 a 2 na realização das tarefas, totalizando no máximo 32 pontos. Escores abaixo de 19 pontos e entre 20 e 24 pontos representam riscos respectivamente alto e moderado de quedas.

O teste de alcance funcional tem como objetivo determinar o quanto o idoso é capaz de se deslocar dentro dos limites de estabilidade anterior e lateral. É empregado para avaliar a estabilidade anteroposterior, medindo o deslocamento máximo anterior com o braço estendido, sem mover os pés.

Os idosos que alcançaram entre $15,2 \mathrm{~cm}$ e 25,3 $\mathrm{cm}$ tiveram um risco duas vezes maior de cair do que aqueles que alcançaram $25,4 \mathrm{~cm}$ ou mais. Os idosos que alcançaram menos que 15,2 cm tiveram quatro vezes mais chance de cair. ${ }^{9}$

\section{Medicamentos e quedas}

Os idosos são os indivíduos que mais consomem medicamentos, sejam prescritos pelo médico ou por automedicação. Teoricamente, qualquer medicamento pode ser causa de instabilidade postural, tonturas ou até mesmo quedas em idosos; por isso, em toda consulta os medicamentos em uso (prescritos ou não) devem ser avaliados, assim como a dose e o tempo de uso. Um agravante entre os idosos é a utilização de muitas drogas ao mesmo tempo (polifarmácia), com o aumento da ocorrência de interações medicamentosas, podendo haver sinergismo ou antagonismo entre elas. ${ }^{10-12}$

\section{Mecanismos das quedas relacionadas às drogas}

Com o envelhecimento, a farmacocinética das drogas pode sofrer alterações, ${ }^{13}$ sendo o termo usado para descrever as etapas desde a administração até a excreção dos medicamentos, a saber: absorção, distribuição, biotransformação e excreção. A redução da secreção de ácido gástrico, assim como a redução do tempo de esvaziamento gástrico, aumenta a degradação da droga, podendo até determinar a inativação de algumas, como a levodopa, por exemplo. Outro problema comum é a interferência de um medicamento na absorção de outro, como ocorre com os antiácidos, que reduzem a absorção da cimetidina e dos derivados imidazólicos. Ocorrem também alterações intestinais, como a aceleração no trânsito intestinal e redução da absorção ou a lentificação do trânsito intestinal com o aumento da absorção. 
Durante o processo de envelhecimento ocorre redução da massa muscular, o tecido adiposo aumenta e, por consequência, a distribuição das drogas lipofílicas também. A água corporal reduz-se, assim como a albumina sérica. Como muitas drogas são hidrossolúveis e há menos água disponível para sua dissolução, essas drogas atingem níveis mais elevados de concentração nas pessoas idosas.

$\mathrm{Na}$ etapa denominada biotransformação ou metabolismo, ocorre a redução da capacidade hepática para inativar substâncias em até $40 \%{ }^{13}$

A última etapa é a excreção, na qual ocorre a redução da filtração glomerular em 35 a 50\%, redução do número de néfrons, aumento do tecido conectivo intersticial e da hialinização glomerular, espessamento da membrana basal, redução do fluxo plasmático renal com o prolongamento do tempo de ação das drogas no organismo e a redução da janela terapêutica.

A farmacodinâmica também está alterada nos idosos, pois eles têm maior sensibilidade a várias drogas decorrente do menor controle postural devido às alterações na barorregulação, dificuldades de termorregulação, aumento da intolerância à glicose, alterações de sensibilidade à ação enzimática e uma resposta imunitária reduzida, particularmente a celular. Todas estas alterações na farmacocinética e farmacodinâmica aumentam a toxicidade das drogas nos idosos.

Diante de tantos desafios à prescrição medicamentosa, devemos ter precauções para evitarmos a iatrogenia farmacológica. A seguir sugerimos algumas condutas que auxiliarão o médico na hora de prescrever um medicamento: evitar usar drogas recém-chegadas ao mercado, pois elas são testadas em indivíduos jovens e saudáveis - os idosos quase sempre são excluídos dos ensaios clínicos; ter muito cuidado com as fórmulas de manipulação, pois elas costumam conter várias substâncias ativas associadas; buscar o diagnóstico preciso exaustivamente, a fim de evitar tratamento de queixas subjetivas como tonteira, indisposição, fadiga, esquecimento; iniciar o tratamento com objetivos definidos e tempo determinado, pois alguns indivíduos usam medicamentos por tempo indeterminado pela falta de orientação correta de quando cessar o uso; usar a menor dose terapêutica possível; ter cuidados especiais com os pacientes deprimidos, com deficit cognitivo, com deficiência visual ou aqueles que vivem sós e precisam cuidar dos próprios remédios; atentar às apresentações em gotas e à necessidade de partir medicamentos; usar o menor número de medicamentos e facilitar ao máximo a posologia.

\section{Alterações da marcha}

Habitualmente as quedas são consequências dos distúrbios do equilíbrio e da marcha, algumas marchas são típicas de patologias específicas e podem auxiliar o médico a elaborar a hipótese diagnóstica mais provável, ${ }^{14-16}$ como as listadas a seguir.

Na marcha frontalizada ou do lobo frontal, ocorre o alargamento da base de suporte e os pés parecem estar grudados no chão como se fossem atraídos por um ímã, de modo que esta marcha é também chamada de magnética. Uma vez iniciada a marcha, a pessoa pode interrompê-la abruptamente. Essa alteração se associa aos estágios avançados da doença de Alzheimer, demências vasculares, doença de Binswanger e hidrocefalia de pressão normal.

Na marcha cerebelar atáxica, a base de suporte fica alargada, os passos são pequenos, irregulares e instáveis, o equilíbrio e o controle sobre o tronco e os movimentos das pernas estão muito prejudicados. Às vezes, os passos são cambaleantes, dando a impressão de indivíduo bêbado, sendo por isso também chamada de marcha ebriosa. As causas mais comuns são: danos vestibulares agudos, acidentes vasculares encefálicos, a lcoolismo crônico, paralisia supranuclear progressiva e atrofia de múltiplos sistemas.

A marcha espástica é causada por hemiplegia, hemiparesia ou paraparesia. O membro afetado é rígido, ligeiramente fletido na bacia e estendido no joelho, e o pé permanece em flexão plantar. O balanço do braço afetado está prejudicado, os dedos do pé afetado arranham ou se arrastam sobre o solo. Para andar, o paciente balança o membro afetado em arco externo a cada passo.

A marcha paraparética ou paraplégica espástica é, na verdade, uma marcha hemiplégica bilateral, que afeta apenas os membros inferiores. Cada perna é avançada lenta e rigidamente, com movimentação restrita nos quadris e joelhos. As pernas encontram-se esticadas ou ligeiramente flexionadas na altura dos joelhos e as coxas po- 
dem estar fortemente aduzidas, fazendo com que as pernas quase se cruzem quando o paciente caminha (marcha em tesoura). As passadas são regulares e curtas, sendo que o paciente avança apenas com grande esforço, como se caminhasse com dificuldade em águas fundas. As principais causas são: mielopatia cervical espondilítica, anemia perniciosa, compressão crônica da coluna espinhal, hipertireoidismo e infartos lacunares.

$\mathrm{Na}$ marcha anserina há queixas de dificuldades de subir escadas e levantar de cadeiras. Causada pela perda da força muscular envolvendo a cintura coxofemoral, provoca uma rotação exagerada da pelve e rolamento da bacia a cada passo. As causas mais comuns são: hipotireoidismo, hipertireoidismo, polimialgia reumática, polimiosite, osteomalacia e neuropatias proximais.

Na marcha festinante, os passos são rápidos e simétricos, o tronco está inclinado para frente, a bacia e joelhos estão mais fletidos. As causas mais comuns são: doença de Parkinson, parkinsonismo, múltiplos infartos cerebrais, demência de Alzheimer, hidrocefalia de pressão normal e demência por corpúsculos de Lewy.
A marcha por deficit multissensoriais é causada por distúrbios dos sistemas visual, proprioceptivo e vestibular. O indivíduo queixa-se de tonteiras, instabilidade, sensação de cabeça vazia quando caminha ou se vira rapidamente. A causa mais comum é o diabetes mellitus.

Na marcha vestibular, a base de suporte está alargada e o indivíduo queixa-se de constante sensação de instabilidade ao caminhar. Há frequentes tropeços laterais, ligeira tendência a caminhar em direção ao lado da disfunção vestibular, seguida de uma correção rápida na direção oposta, condição que piora quando os olhos estão fechados. As causas mais comuns são: labirintopatias, toxicidade por drogas (aminoglicosídeos) e tumores do ângulo pontocerebelar.

A marcha antálgica é causada por processos inflamatórios articulares crônicos ou agudos, patologias dos pés e articulações afins.

Finalmente, na marcha sensorial atáxica, a base de suporte está alargada e os pés arrastam no chão, as pernas são movimentadas para frente e para fora, em passos com os pés levantados. As causas mais comuns são: doenças do corno

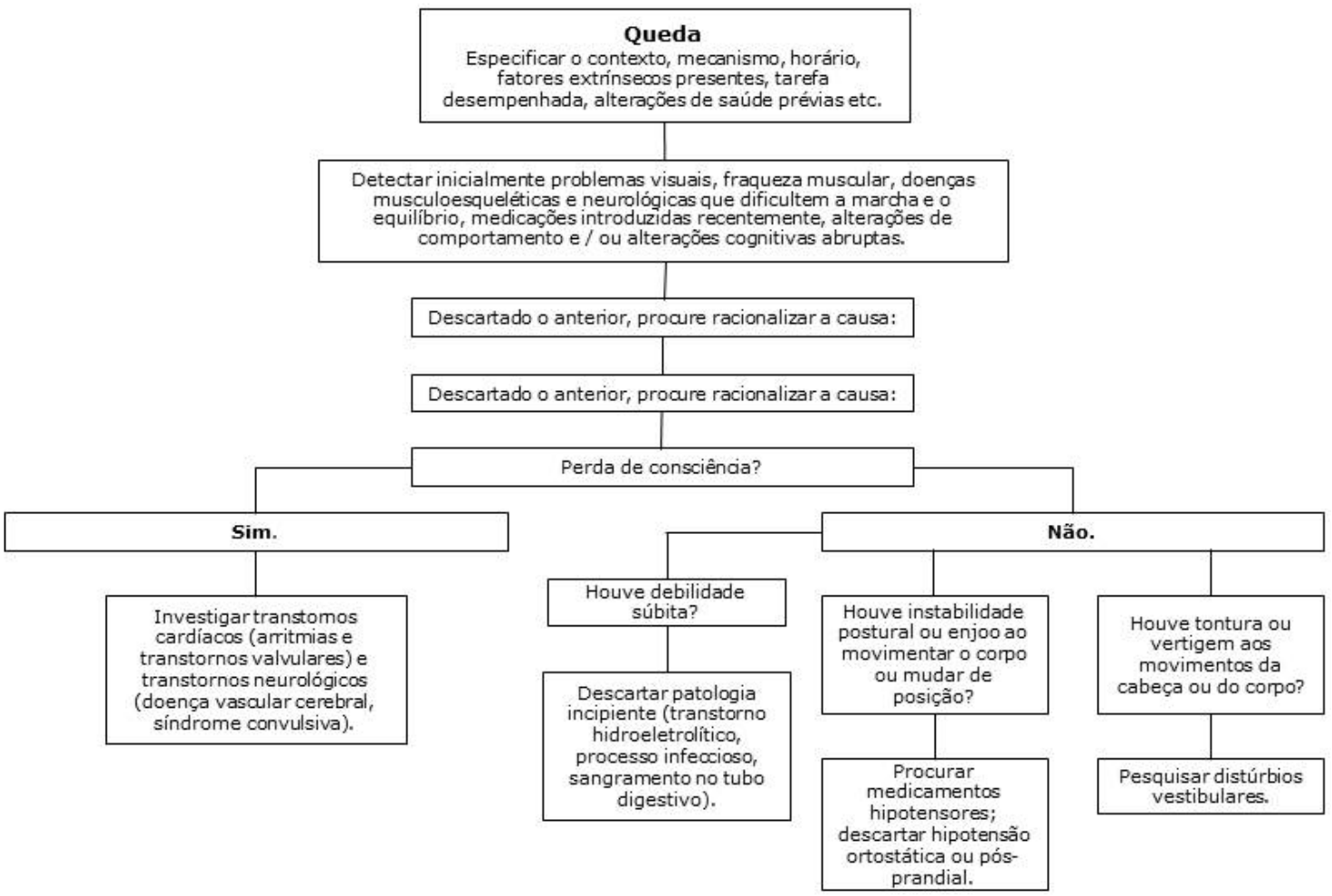

Figura 1. A figura mostra um algoritmo proposto pela OPAS, 2002 para nortear a condução dos casos de quedas em idoso. ${ }^{19}$ 
Quadro 1. Fatores de risco para quedas em idosos e intervenções propostas

\begin{tabular}{|c|c|}
\hline Fatores de risco para quedas & Intervenções \\
\hline $\begin{array}{l}\text { Fraqueza muscular de MMII, dificuldade de levantar da } \\
\text { cadeira }\end{array}$ & $\begin{array}{l}\text { Avaliação nutricional, programa de fortalecimento muscular } \\
\text { de quadríceps e dorsiflexores de tornozelo }\end{array}$ \\
\hline Distúrbio do equilíbrio & $\begin{array}{l}\text { Tratamento das patologias específicas } \\
\text { Adequação da medicação em uso } \\
\text { Treino de equilíbrio }\end{array}$ \\
\hline Distúrbios da marcha & $\begin{array}{l}\text { Tratamento das patologias específicas } \\
\text { Adequação da medicação em uso } \\
\text { Adequação e/ou prescrição de dispositivos de auxílio à marcha } \\
\text { Uso de protetores de quadril } \\
\text { Visita ao podólogo } \\
\text { Uso de sapatos adequados }\end{array}$ \\
\hline Deficit visual & $\begin{array}{l}\text { Visita ao oftalmologista } \\
\text { Adequação de lentes corretivas } \\
\text { Evitar o uso de lentes bifocais }\end{array}$ \\
\hline Deficit auditivo & $\begin{array}{l}\text { Prescrição e uso adequado do aparelho de amplificação } \\
\text { sonora }\end{array}$ \\
\hline Hipotensão ortostática & $\begin{array}{l}\text { Revisão da medicação, elevação da cabeceira da cama, } \\
\text { orientação de movimentos de MMIl antes de se levantar, } \\
\text { exclusão da desidratação, avaliação do uso de sal e acetato de } \\
\text { fludrocortisona }\end{array}$ \\
\hline Uso de medicações psicotrópicas & Revisão da necessidade de uso \\
\hline Presença de riscos ambientais & Modificação ambiental e adaptações necessárias \\
\hline Queixa de tontura & Se necessário, encaminhamento ao otoneurologista. \\
\hline Agitação psicomotora, confusão mental & $\begin{array}{l}\text { Avaliação da presença de quadro de estado confusional agudo } \\
\text { Adequação do ciclo vigília-sono } \\
\text { Implementação de medidas de higiene do sono } \\
\text { Evitar eventos estressores } \\
\text { Evitar restrição física ou medicamentosa } \\
\text { Vigilância contínua. }\end{array}$ \\
\hline $\begin{array}{l}\text { Doença de Parkinson, parkinsonismo, acidente vascular en- } \\
\text { cefálico, artrite, neuropatias, demência }\end{array}$ & $\begin{array}{l}\text { Manejo farmacológico específico } \\
\text { Fisioterapia especializada }\end{array}$ \\
\hline
\end{tabular}

posterior da coluna e neuropatia periférica.

\section{Exames complementares}

Não existe nenhum exame complementar específico para o diagnóstico dos distúrbios da marcha. Eles devem ser solicitados de acordo com as hipóteses diagnósticas elaboradas após a anamnese e o exame físico.

A densitometria óssea deve fazer parte da investigação do idoso com risco de cair para o diagnóstico da osteoporose.

\section{Condutas}

Após a minuciosa avaliação de todos os fatores que estão contribuindo para as quedas, habi- tualmente mais de uma causa pode ser detectada; o médico deve formular uma lista dos problemas identificados e elaborar um plano de tratamento e acompanhamento dos resultados. ${ }^{17}$

As intervenções devem ser realizadas com o intuito de prevenir a primeira queda nos indivíduos que começaram a apresentar alterações da marcha recentemente ou diminuir o risco de novas quedas naqueles que já caíram. Inicialmente todas as doenças diagnosticadas devem ser tratadas e os deficit sensoriais devem ser corrigidos, assim como os problemas nos pés.

Uma revisão e adequação medicamentosa é fundamental. A suspensão dos medicamentos não essenciais deve ser feita, assim como a redução das doses daqueles que estiverem na dosagem maior que a necessária. Alguns medicamentos 
com indicação duvidosa também devem ser suspensos.

Um programa de exercícios adequados para cada paciente deve ser indicado e o uso de auxiliares da marcha deve ser avaliado nos casos mais graves. ${ }^{18}$

Alguns pacientes com alto risco de quedas devem ser orientados a não andarem sozinhos; seus familiares devem saber o risco e as consequências das quedas e ser incentivados a impedir que o idoso ande sozinho. O quadro 1 mostra alguns fatores de risco para quedas em idosos e sugere algumas intervenções que podem reduzir sua ocorrência.

\section{Conclusões}

O médico tem um papel fundamental na avaliação do idoso que cai, assim como daqueles que não caíram, mas que estão com alterações em seu equilíbrio e marcha e, consequentemente, correm o risco de cair. A investigação exaustiva da etiologia das quedas, assim como uma busca ativa dos fatores de risco para a ocorrência de uma futura queda, é papel do médico que se propõe a cuidar de pacientes idosos.

Os idosos não estão predestinados a cair e nem mesmo andar com dificuldade. Eles devem receber avaliações periódicas de profissionais capacitados e instruções que os auxiliem a manter uma marcha segura mesmo em idades avançadas.

\section{Referências}

1. Oliver D. Older people who fall: why they matter and what you can do. Br J Community Nurs. 2007;12(11):500-7.

2. Callisaya ML, Blizzard L, Schmidt MD, McGinley JL, Lord SR, Srikanth VK. A population-based study of sensorimotor factors affecting gait in older people. Age Ageing. 2009;38(3):290-5.

3. Mader SL. Identification and management of orthostatic hypotension in older and medically complex patients. Expert Rev Cardiovasc Ther. 2012;10(3):38795.

4. Snijders AH, van de Warrenburg BP, Giladi N, Bloem BR. Neurological gait disorders in elderly people: clinical approach and classification. Lancet Neurol. 2007;6(1):63-74.

5. Leveille SG, Jones RN, Kiely DK, Hausdorff JM, Shmerling RH, Guralnik JM, et al. Chronic musculoskeletal pain and the occurrence of falls in an older population. JAMA. 2009;302(20):2214-21.

6. Perracini MR, Ramos LR. Fatores associados a quedas em uma coorte de idosos residentes na comunidade. Rev Saúde Pública. 2002;36(6):709-16.

7. Mathias S, Nayak US, Isaacs B. Balance in elderly patients: the "get-up and go" test. Arch Phys Med Rehabil. 1986;67(6):387-9.

8. Tinetti ME. Performance-oriented assessment of mobility problems in elderly patients. J Am Geriatr Soc. 1986;34(2):119-26.

9. Duncan PW, Weiner DK, Chandler J, Studenski S. Functional reach: a new clinical measure of balance. J Gerontol. 1990;45(6):M192-7.

10. Lau DT, Mercaldo ND, Shega JW, Rademaker A, Weintraub S. Functional decline associated with polypharmacy and potentially inappropriate medications in community-dwelling older adults with dementia. Am J Alzheimers Dis Other Demen. 2011;26(8):606-15.

11. American Geriatrics Society 2012 Beers Criteria Update Expert Panel. American Geriatrics Society updated Beers Criteria for potentially inappropriate medication use in older adults. J Am Geriatr Soc. 2012;60(4):616-31.

12. Hill KD, Wee R. Psychotropic drug-induced falls in older people: a review of interventions aimed at reducing the problem. Drugs Aging. 2012;29(1):15-30.

13. Sera LC, McPherson ML. Pharmacokinetics and pharmacodynamic changes associated with aging and implications for drug therapy. Clin Geriatr Med. 2012;28(2):273-86.

14. Beauchet O, Allali G, Annweiler C, Bridenbaugh S, Assal F, Kressig RW, et al. Gait variability among healthy adults: low and high stride-to-stride variability are both a reflection of gait stability. Gerontology. 2009;55(6):702-6

15. Ferrucci L, Bandinelli S, Benvenuti E, Di lorio A, Macchi C, Harris TB, et al. Subsystems contributing to the decline in ability to walk: bridging the gap between epidemiology and geriatric practice in the InCHIANTI Study. J Am Geriatr Soc. 2000;48(12):161825.

16. Kirkwood RN, de Souza Moreira B, Vallone ML, Mingoti SA, Dias RC, Sampaio RF. Step length to be a strong discriminant gait parameter for elderly females highly concerned about falls: a cross-sectional observational study. Physiotherapy. 2011;97(2):126-31.

17. Tinetti ME, Baker DI, McAvay G, Claus EB, Garrett P, Gottschalk M, et al. A multifactorial intervention to reduce the risk of falling among elderly people living in the community. N Engl J Med. 1994;331(13):821-7.

18. Merom D, Pye V, Macniven R, van der Ploeg H, Milat A, Sherrington $C$, et al. Prevalence and correlates of participation in fall prevention exercise/physical 
Revista HUPE, Rio de Janeiro, 2014;13(2):45-52

activity by older adults. Prev Med. 2012;55(6):613-7.

19. Organización Panamericana de la Salud. OPAS. Guia clínica para atención primaria a las personas adultas mayores. Washington DC: OPAS; 2003

Recebido: 08/03/2013.

Revisado: 26/10/2013.

Aprovado: 06/12/2013.

\section{Emylucy M. P. Paradela}

Departamento de Medicina Interna. Faculdade de Ciências Médicas. Universidade do Estado do Rio de Janeiro. Rio de Janeiro, RJ, Brasil. 